\title{
Game of Drones
}

Joseph Lindley

HighWire CDT

Lancaster University

joseph.lindley@gmail.com

\section{Paul Coulton}

Imagination

Lancaster University

p.coulton@lancaster.ac.uk

\section{Abstract}

In response to the recent European Directive the UK government sanctioned the use of drones by

commercial providers subject to pilots holding an approved Drone Pilot Proficiency Certificate (DPPC). As the government anticipated the main use has been in providing services to local authorities that aid in the enforcement of local by-laws. Whilst many commercial providers have followed the traditional path of employing dedicated enforcement officers to pilot the drones, in this paper we present on-going research that 'gamifies' the enforcment activities to allow members of the local community to act as enforcement officers. In particular we have worked with retired members of the police and armed services as drone pilots in relation to the enforcement of by-laws relating to parking offences and dog fouling in a small UK city. The initial results indicate that not only does this age group find the game-like activity enjoyable they feel that they are providing an important service to their community.

\section{Author Keywords}

Game design; privacy; drones; playbour; design fiction.

\section{ACM Classification Keywords}

I.2.9. Robotics: Operator Interfaces.

\section{Introduction}

While they were once the exclusive preserve of the military the adoption of Unmanned Aerial Vehicles 


\section{CPA 658 Article 167}

stated that aircraft must not be flown:

- $\quad$ at a height less than 122 metres;

- over or within 150

metres of any congested area

- $\quad$ over or within 150

metres of an organised open-

air assembly of more than

1,000 persons;

- within 50 metres of any

vessel, vehicle or structure

which is not under the control

of the person in charge of the aircraft

- within 50 metres of any person except during take-off or landing, the aircraft must not be flown within 30 metres of any person except for the person in charge of the aircraft.
(UAV's), commonly referred to as drones, in a wide variety of civilian applications, such as Amazon Prime Air $\AA$ and Google Follow $\AA$, means they have become a common sight in many cities around the world.

In the UK the adoption of commercial services has been limited due to these small commercial drones being subject to the Civil Aviation Authority Protocol: Model Aircraft: A Guide to Safe Flying (CAP 658) [1]. The drones within this research were previously subject to Article 167 - Small unmanned surveillance aircraft, as they are less than $7 \mathrm{Kg}$ in weight.

A further limitation was that First Person View (FPV) Radio Control (R/C) required the operation of a Buddy Box system which stipulated the person in charge of the UAV holds the master transmitter and must maintain direct unaided visual contact with the UAV whilst another person flies the aircraft by reference to the live video from the on-board camera.

Thus the new legislation requires commercial drones to be fitted with sensors such that their location, direction and altitude is monitored in real-time. Dependent upon their operation purpose, distance from base, and height restrictions are enforced centrally and automatically by the system.

In this research we are concerned with drones covered by operational use Type 2 of Article 168, which supersedes pervious article 167 for commercial operation, and states that aircraft must not be flown:

- if the service operator is a not designated provider of the local authority;
- by any person under 18 years of age and not validated to do so with current Drone Pilot Proficiency Certificate (DPPC);

- at a height below 4.5 metres (typical lamppost height in UK) unless landing at a designated drone station;

- at a distance of over 500 metres from the designated drone station unless returning to central operating station;

- in weather conditions deemed unsuitable on that day by local authority;

- In areas not previously approved by local authority audit.

There are additional regulations relating to safety features of the drone itself but that specific legislation is beyond the scope of this paper.

Given these changes commercial drone operation is now viable within the UK which has led to the emergence of a number of companies offering drone based services. This research pertains to a commercial provider whose drone system facilitates the enforcement of local by-laws relating to parking offences and dog fouling.

While the vast majority of commercial providers have taken a traditional approach, employing dedicated enforcement officers to pilot the drones, in this paper we present on-going research that allows members of the local community to pilot the drones and thus act as enforcement officers. In particular it explores the gamification of these enforcement activities so that they are seen not only as important but one that can bring direct benefit to members of the local community. 


\section{Drone Hardware}

The drone used is a 3DR Solo fitted with a 3 Axis gimbal housing a GoPro Hero 4 camera. The drone also uses the Ballistic Parachute module to meet the safety requirements of flying over populated areas

Each drone uses a lithium polymer battery, which supports a 30 minute flight time on a single charge, and charging at $5 \mathrm{C}$ takes a maximum of 1 hour.
In the following section we describe the system infrastructure as currently deployed.

\section{Drone Enforcement System}

\section{Drone Infrastructure}

The main element of the Drone Enforcement System (DES) is the drone docking station shown in Figure 1. The docking station takes advantage of a streetlamp design which incorporates reflectors (to significantly increase the amount of light delivered) [3] - these provide a convenient landing stations for the enforcement drones.

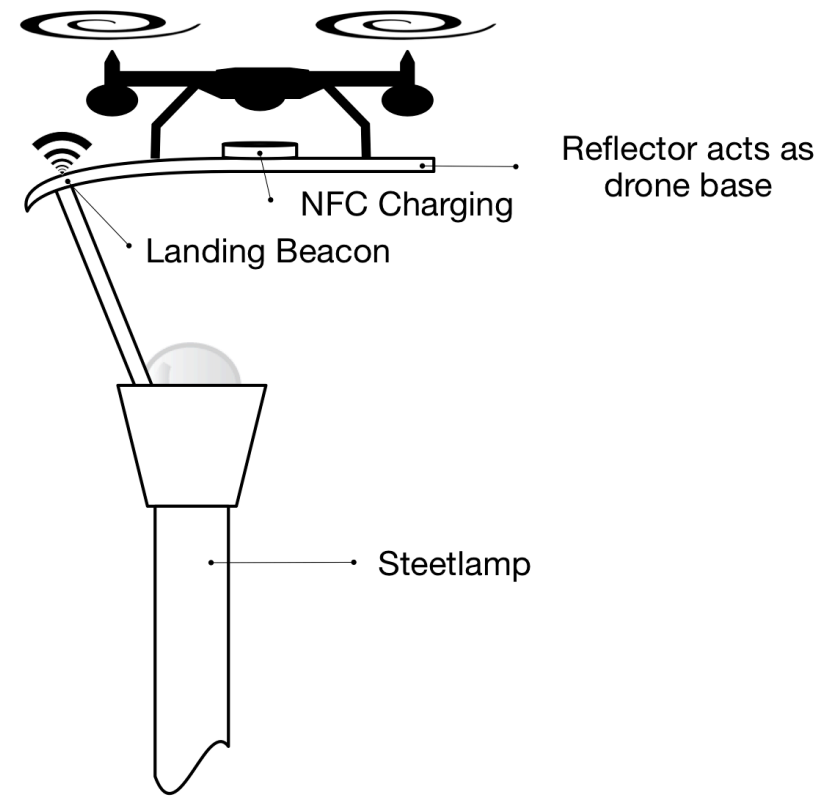

Figure 1. Drone Docking Station.
To create the docking station we added a number of additional features to the reflector base. The first is a Near Field Communications (NFC) enabled wireless charging unit, which allows the drone battery to be wirelessly charged [4] in-between flights. The second feature is a beacon to allow automated precision landing atop the docking station. This also means at the start and end of the enforcement period each day the system can automatically fly the drone to and from the operating system using GPS to provide navigation and use the beacon the provide the positional granularity required to land the drone safely.

In parking enforcement mode the system uses image recognition software to identify both the number plate of the vehicle and the parking zone permit displayed in the windscreen. In the current version of the system while visitor parking permits can be indentified the software cannot reliably identify the handwritten details for a particular day, therefore the system alerts a human enforcement officer to check the vehicle by sending its location and vehicle registration number.

The alternate enforcement activity relating to dog fouling was specifically chosen because it does not naturally lend itself to automation through image processing and is therefore highly dependent on the human operator to both monitor the dog fouling, and monitor whether owners take appropriate action when fouling occurs. As all drone footage is recorded the operator simply presses a button to mark where the offence appears in the footage. In the initial trials no action was taken, but the intention is for the recorded video clip to be sent to a dog warden. Given the recent advances in facial recognition this could form part of a future enforcement system. 
Pilot Control

When logging in to the system users are presented with a list of locations at which drones are currently available, the type of enforcement currently required in that area, and the available flight time of the drone based on current battery level.

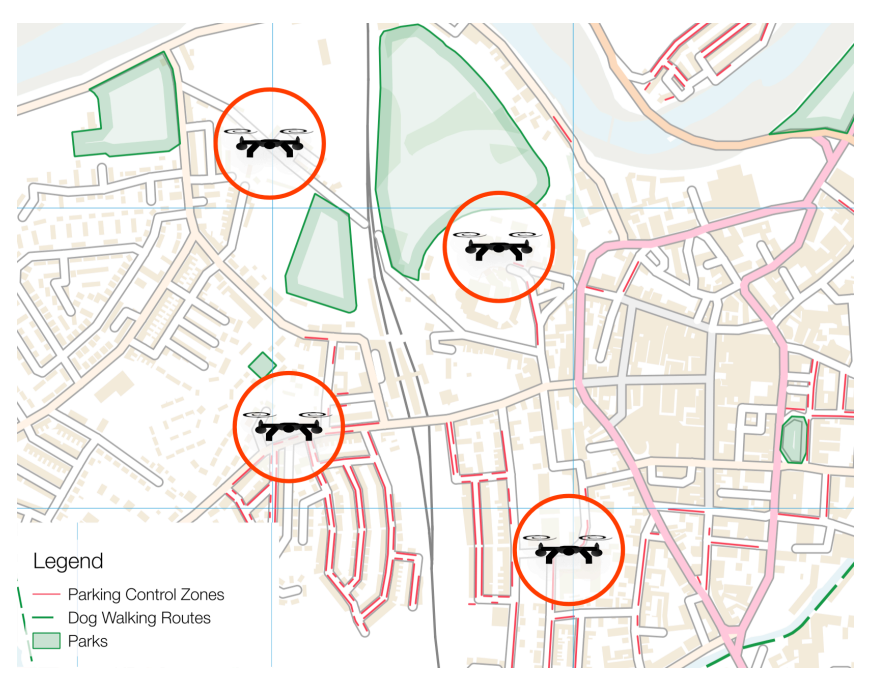

Figure 2. Map of Drone Enforcement Trial Area and Drone Stations

\section{Gamification of Drone Operation}

Whilst gamification is a still a controversial topic [2] it was considered an appropriate approach for providing feedback to the users in regard their performance within the system as there is already a game like aesthetic to the system and the controls for the drone naturally lend themselves to the utilisation of commercial game controllers as shown in Figure 3. Whilst the drone does come with it own controller it was easier create bespoke controls by using a separate controller which connects to a laptop running the system software via Bluetooth LE.

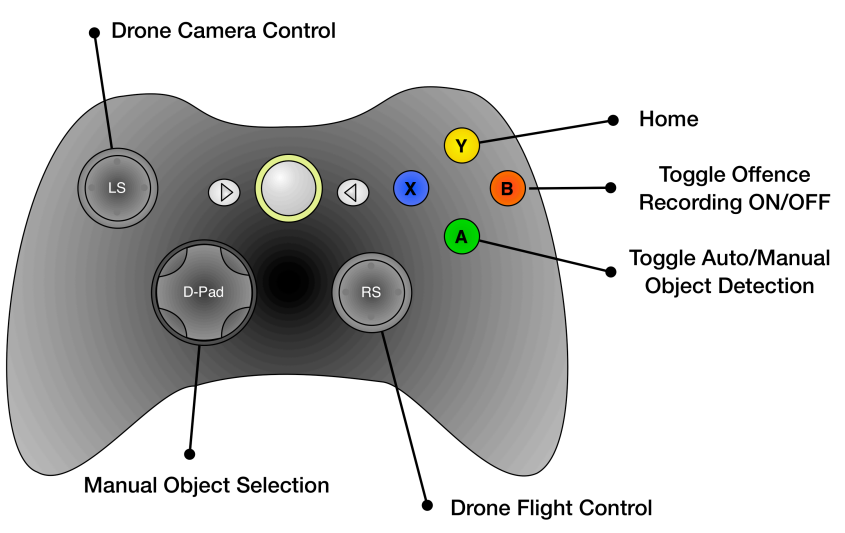

Figure 3. Drone Controls.

The most utilised feedback system for games are points that are principally used to provide a direct indication that some goal within a game has been achieved. In the case of parking enforcement points are awarded for each vehicle checked within extra points awarded for identification of parking offences. A ranking system was also incorporated based on the flight hours recorded by the drone pilots and enforcement points. During dog fouling enforcement points are awarded based on time as it relies solely on the observational skills of the pilot and extra points awarded for successful detection of an offence.

There was a great deal of debate within the project team as to whether awarding points directly might lead to accusations by the public of promoting over zealous enforcement, however, it was decided that this would 
only be an issue if payment was related to this aspect of performance.

\section{Drone Trials}

The preliminary trials of the DES system was initially trialed by four individuals, 3 former military and one former police personnel, who were able to pilot any of the four drones located at sites shown in Figure 2.
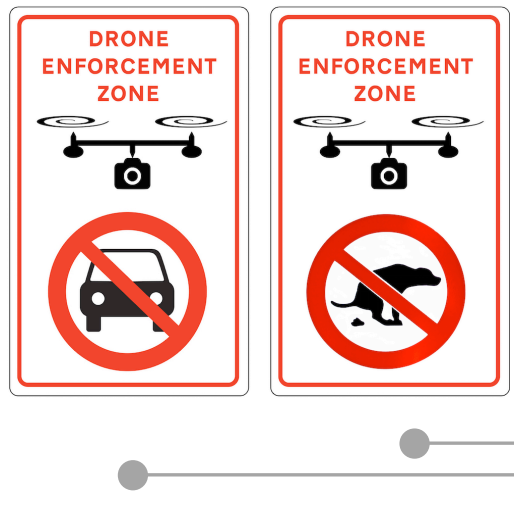

O
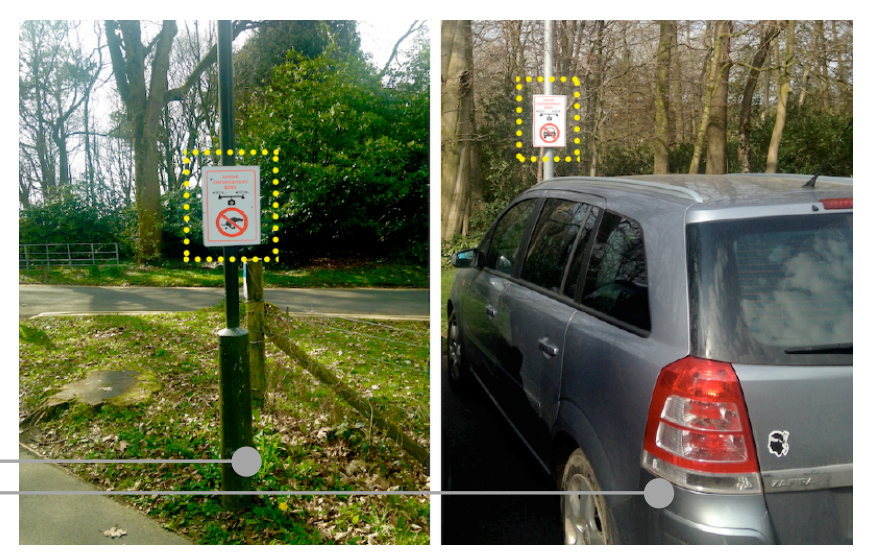

Figure 4. Drone Enforcement Notices (denoted by yellow box).

These sites were chosen specifically to allow both enforcement activities to be performed. Each site had a programmatically enforced boundary of $500 \mathrm{~m}$ and a $5 \mathrm{~m}$ no-fly zone was enforced in relation to the train line that can be seen running up the centre of the map. The zones were highlighted to the general public through the installation of drone enforcement notices as shown in Figure 4.

The initial trial was designed to evaluate the system's command and control infrastructure, the usability of the interface, and generate preliminary insights into the effectiveness, impact and feasibility of drone enforcement. Two enforcement applications were included in the initial trial and all of the participants were given statutory training to DPPC level as well as two days training on specifically on the DES.

While the initial systems trial only involved four drones to accommodate the eventuality that all four trial participants may simultaneously be working within the DES for extended periods of time during peak activity a total of 16 drones will be used in the next stage of the project.

Several types of data were gathered in order to provide an overview of the DES's usability and effectiveness. This includes a range of flight telemetry (flight time, distance covered, speed, height etc) as well as data directly relating to enforcement applications. These data primarily focus on the identification of 'targets': number of targets identified; targets identified but not confirmed by the DES; activities logged manually by the operator. All flights are fully video recorded, and archived in accordance with data protection laws prescribed by the Information Commissioner's Office (ICO) which are the same regulations applied to CCTV footage. In addition to the recording of dog fouling incidents the drone pilots are also encouraged to record any activity they consider 'unusual' to ascertain of the use of drones has potential for crime prevention beyond enforcement activities.

Alongside system-generated data, trial participants are encouraged to keep regular reflective logs and were required to attend a half day debrief discussion session at the end of the trial. The usability and effectiveness study is based upon these data sets: telemetry, system 

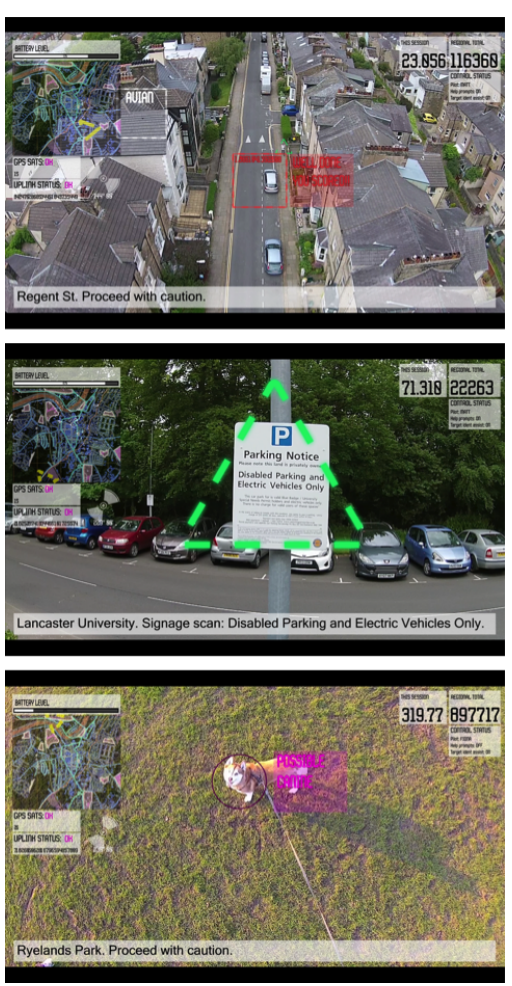

Figure 5. Screenshots captured during DES trails generated data, curious incident videos, daily logs and the debrief discussion.

To assess the appeal of the gamified aspects of enforcement system, we did not specify how much or little the DES should be used during the trial, so long as participants used the DES for a minimum of 60 minutes per day, did not exceed the maximum single session duration (240 minutes), and kept within operational parameters for daylight and weather conditions. During the feedback sessions the operators we asked to reflect on the feedback systems and consider whether specific operating targets would be useful additions to the system.

Whilst the data generated has been considerable, as shown in the screenshots (figure 5) and will require further analysis along with more extensive trials, the initial results indicate that not only does this age group find the game-like activity enjoyable they feel that they are providing an important role within their community.

\section{Conclusions}

The research in this paper and the associated artifacts are part of a design fiction [5]. Therefore, whilst this paper presents a fictional account of plausible future $\mathrm{HCI}$ research its purpose is not only to highlight potential usability or utility issues such systems might present but to also create a discursive space in which researchers can consider the wider societal and ethical issues of technological futures in which drones might be widely adopted. In future publications we will consider the effectiveness of this design fiction in addressing such challenges and design fiction more generally as a method for exploring issues related to introduction of technologies.

\section{Supporting Video}

Please see http://youtu.be/6b 30d7yW2s to access actual archived (design fiction) footage of the DES in use.

\section{Acknowledgements}

Thank you to Stephen Edwards, Andy Darby, and Moog (the dog) for agreeing to feature in the video. This work was supported by RCUK Digital Economy Programme (Grant Reference EP/G037582/1) and was carried at the HighWire Centre for Doctoral Training and Imagination Lancaster.

\section{References}

[1] Civil Aviation Authority. CAP 658 Model Aircraft: A Guide to Safe Flying.

http://www.caa.co.uk/docs/33/CAP658\%204\%20Editio n\%20Amend\%201\%20June\%202013.pdf.

[2] Deterding, S. (2015). The Ambiguity of Games: Histories and Discourses of a Gameful World, in The Gameful World, (pp 23-64), MIT Press.

[3] Elmer, W. B. (1966). A study in street lighting reflector design. Applied optics, 5(2), 343-349.

[4] Strommer, E., Jurvansuu, M., Tuikka, T., Ylisaukkooja, A., Rapakko, H., \& Vesterinen, J. (2012, March). NFC-enabled wireless charging. In Near Field Communication (NFC), 2012 4th International Workshop on (pp. 36-41). IEEE.

[5] Lindley, J., \& Coulton, P. (2015). Back to the future: 10 years of design fiction. In Proceedings of 2015 British Human Computer Interaction Conference. 